Archives de sciences sociales des religions

113 | janvier-mars 2001

Varia

\title{
La Vierge de Tinos : le cœur sacré de l'État grec
}

\section{Katerina Seraïdari}

\section{OpenEdition}

Journals

Édition électronique

URL : http://journals.openedition.org/assr/20186

DOI : 10.4000/assr.20186

ISSN : $1777-5825$

Éditeur

Éditions de l'EHESS

Édition imprimée

Date de publication : 1 avril 2001

Pagination : 45-59

ISBN : 2-222-96701-5

ISSN : 0335-5985

Référence électronique

Katerina Seraïdari, «La Vierge de Tinos : le cœur sacré de l'État grec », Archives de sciences sociales des religions [En ligne], 113 | janvier-mars 2001, mis en ligne le 19 août 2009, consulté le 19 avril 2019 URL : http://journals.openedition.org/assr/20186 ; DOI : 10.4000/assr.20186

Ce document a été généré automatiquement le 19 avril 2019

(c) Archives de sciences sociales des religions 


\title{
La Vierge de Tinos : le cœur sacré de l'État grec
}

\author{
Katerina Seraïdari
}

1 La révolution grecque ${ }^{1}$ contre les Ottomans, qui dominaient le pays depuis quatre siècles, commence, selon l'histoire officielle, le 25 mars 1821. Selon la légende de Tinos, une île des Cyclades où se trouve l'un des sanctuaires mariaux les plus connus de la Grèce, c'est quelques jours avant cette date que l'icône qui y est vénérée commença à manifester sa volonté d'être rendue au culte : cette icône, représentant l'Annonciation, était enterrée à l'endroit où s'élève son sanctuaire depuis 1200 , date où les Sarrasins auraient brûlé l'église qui l'abritait depuis les premiers siècles du christianisme.

2 La coïncidence de ces deux "événements " n'a évidemment rien de fortuit. La nation grecque s'est construite en luttant contre un pouvoir musulman. Il n'est donc pas étonnant qu'elle ait valorisé la dimension chrétienne de son identité : aussi a-t-elle choisi de dater le début de sa lutte pour l'indépendance du 25 mars, jour de l'Annonciation de la Vierge. Il est clair, à l'inverse, que les auteurs de la légende de Tinos ont voulu inscrire l'« invention ${ }^{2}$ » de son icône dans l'histoire de la révolution, articulant ainsi l'histoire du sanctuaire et l'histoire nationale. L'opération a, dans un premier temps, parfaitement réussi. Le nouvel État, on le verra, a reconnu la dimension nationale du sanctuaire et celui-ci est arrivé à s'imposer très tôt sur les autres lieux de pèlerinage plus anciens du territoire et à établir sa réputation pan-hellénique.

3 L'accord entre le nationalisme et la religion, cependant, ne fut pas toujours parfait et le cas de Tinos le montre de façon exemplaire. La construction nationale s'est appuyée sur le sentiment et les symboles religieux ainsi que sur les structures ecclésiastiques pour se légitimer, tout en rappelant, à l'occasion, son autonomie. Ainsi, en 1833 et durant une période de dix-sept ans, le roi Othon mit en place une politique de séparation de l'Église et de l'État et décida de supprimer les trois quart des monastères grecs. Dans la même période, l'Église grecque devint autonome, ne dépendant plus du Patriarche de Constantinople. Néanmoins, par la suite, la séparation de l'Église et de l'État et l'autonomie vis-à-vis de Constantinople ont été révisées et abandonnées. 
4 Le statut de l'Église de Tinos, on le verra, constitue aujourd'hui un cas particulier, parce qu'il reflète l'esprit de réforme de l'époque d'Othon. L'Église officielle n'a cessé de demander le contrôle sur l'Institution qui régit le sanctuaire de Tinos et le changement de son statut exceptionnel. Ainsi, par exemple, entre 1903 et 1908, l'évêque de l'île prit des mesures radicales afin de l'obliger à se soumettre à l'Église et ordonna aux popes qui officiaient de ne plus servir la messe. Plus récemment, un ecclésiastique ${ }^{3}$ accusa les membres civils de l'Institution de dépenser l'argent afin de manipuler les votes et de favoriser l'élection de certains députés de l'île au Parlement, c'est-à-dire de se mêler à la vie politique du pays; ce même clerc affirma qu'à l'occasion de l'élection de députés favorables à l'Institution, l'église de Tinos fut décorée comme si c'était un jour de fête religieuse.

Entre le religieux et le politique, la piété individuelle et le nationalisme, Tinos constitue depuis presque deux siècles un symbole puissant pour le pays et ses habitants. C'est son histoire et son évolution que j'essaierai de retracer dans cet article.

\section{Formation d'un État, invention d'une icône}

6 L'histoire de l'icône de Tinos est racontée dans les brochures, éditées et diffusées par le sanctuaire, qui constitueront une de mes sources essentielles ${ }^{4}$. Cette histoire s'attache à mettre en accord les péripéties de l'icône et les troubles politiques que la nation a subis. Ainsi, en 1204, Constantinople est prise par les croisés qui se partagent ensuite l'empire byzantin. En 1453, Constantinople tombe aux mains des Ottomans. Suivent quatre siècles de domination de la Grèce par un peuple qualifié de «barbare». L'histoire nationale insiste sur la «barbarie » de l'occupation ottomane, tandis que, selon elle, les régions occupées par les Vénitiens ont connu un traitement plus « civilisé ». C'est le cas de Tinos : en 1207, les Vénitiens occupent l'île qui sera la dernière île cycladique à passer sous occupation ottomane en 1715. Quant à l'icône, elle est placée initialement dans une église de l'île dédiée à saint Jean-Baptiste. Cette église n'échappe pas à la fureur des Sarrasins qui la brûlent en 1200. L'icône restera enterrée dans les ruines de son sanctuaire jusqu'à sa découverte. Comme la nation, l'icône est donc victime de l'agression musulmane; l'occupation vénitienne, en revanche, ne la touche pas, puisqu'elle est déjà enfouie. De cette façon, l'histoire de l'icône semble suivre les lignes principales de l'histoire nationale plutôt que celles de l'histoire locale.

7 La juxtaposition de ces chronologies permet aux écrivains anonymes des livrets d'associer le destin de la nation grecque à celui de l'icône: la première soumise, la deuxième enterrée, toutes les deux en attente de leur résurrection. Comme Loring Danforth (1995, p. 15, note 4) l'a écrit, "l'image de la nation "qui se réveille de son sommeil" est très courante dans les idéologies nationalistes ». Cette image est aussi très courante dans les multiples légendes grecques d'invention d'icônes enfouies. Pourtant, l'icône de Tinos est la seule dont le « réveil » préfigure la naissance de la nation. Ainsi, c'est au moment où les Grecs prennent les armes et se lancent dans une série de batailles successives afin d'obtenir leur émancipation politique, que la Vierge apparaît en rêve à un homme âgé de 80 ans, puis à une religieuse, afin de demander l'exhumation de son icône. Les fouilles commencent en septembre 1822. À l'instar de la nation rebellée, la communauté locale prend alors ses outils et s'engage dans une autre sorte de "bataille", qui aboutira à l'invention de l'icône $e^{5}$. Le 30 janvier 1823, à 14 heures, la pioche d'un ouvrier fend en deux une planche de bois : c'est l'icône de la Panayia ${ }^{6}$. Ainsi commence l'histoire du plus grand 
pèlerinage de la Grèce contemporaine même si la Grèce ne commence d'exister comme État que quelques années plus tard, en 1827.

8 Les deux versions de l'histoire, cependant, ne concordent pas exactement. Ainsi, les manuels scolaires ne disent aucun mot de la religieuse de Tinos (canonisée, elle est aujourd'hui la seule sainte originaire de Tinos) ni de l'invention de l'icône. Au contraire, les imprimés diffusés à Tinos se réfèrent à l'impact que la découverte de l'icône aurait eu sur les héros de la révolution. Selon eux, cet événement a été perçu par les révolutionnaires comme l'expression matérielle de la grâce divine et l'« Annonciation » de la réussite de leur lutte. Ce seront les premiers pèlerins célèbres à visiter l'île et l'icône miraculeuse de la Vierge.

9 Une péripétie de la légende d'invention, rapportée dans un ouvrage pieux ${ }^{7}$, va dans le même sens. Les fouilles pour le déterrement de l'icône sont arrêtées par la femme du propriétaire du domaine indiqué qui refuse de donner sa permission. Cette fois, ce n'est pas la Vierge qui intervient pour la faire changer d'avis, mais un révolutionnaire grec qui lui apparaît en rêve et la menace. À son réveil, la femme terrifiée consent à ce que les opérations débutent. Cet épisode illustre bien l'interdépendance de l'invention et de la révolution, de l'icône et de la nation grecque : l'absence de l'une annule l'existence de l'autre. Ainsi, la découverte de l'icône encourage la poursuite de la lutte pour la liberté de la nation et préfigure la victoire finale. De façon analogue, l'intervention d'un révolutionnaire constitue la condition sine qua non pour la «libération » de l'icône enfouie.

10 Alors que les manuels édités par l'État ignorent l'invention de l'icône, les brochures de Tinos mettent l'accent sur la signification de cette invention pour la révolution. Chaque acteur défend ainsi son territoire : pour l'histoire nationale qui veut garder ses distances par rapport au miraculeux, l'invention de l'icône constitue un détail dont on peut se passer ; l'histoire de l'icône, en revanche, s'approprie la structure de l'histoire nationale afin de montrer que celle-ci est gouvernée par la volonté divine. Étant donné que le lieu principal où cette volonté divine se manifeste est l'église de Tinos, il semble que la manipulation de l'histoire nationale contribue à assurer la position centrale de Tinos dans le nouvel État.

11 La construction idéologique diffusée par le sanctuaire de Tinos a très vite atteint son but. En 1827, l'Angleterre, la France et la Russie garantissaient l'indépendance du nouvel État grec; en 1832, Othon de Bavière, d'origine allemande et catholique, fut imposé par ces trois puissances, comme roi de Grèce. Un an plus tard, Othon arriva en Grèce et visita aussitôt l'île de Tinos pour rendre hommage à l'icône vénérée par le peuple sur lequel il était appelé à régner. Par ce geste, il consacrait ce symbole religieux. Mais, ce faisant, il légitimait aussi son pouvoir, imposé par des puissances étrangères, en le mettant sous la protection d'une Vierge « autochtone ». C'était la première fois que l'icône de Tinos était reconnue officiellement comme « icône nationale ».

12 Cependant, ni l'invention pour l'icône ni la formation de l'État pour le nationalisme grec ne sont considérées comme des commencements absolus. Pour tous les deux, le premier besoin à satisfaire est la construction d'un passé continu et signifiant. Par conséquent, deux qualités principales sont mises aussitôt en valeur : l'autochtonie et le fait que leur origine remonte à l'aube des temps. Ainsi, l'histoire diffusée par l'Éducation Nationale explique-t-elle que la «nation grecque » existe dès l'Antiquité. De façon analogue, les imprimés de Tinos affirment que l'icône est une des trois premières icônes que saint Luc peignit en prenant comme modèle la Vierge elle-même. De même que la nation grecque 
se présente comme l'héritière de la civilisation antique qui marque le début de l'histoire européenne, l'icône de Tinos, peinte, selon la légende, par saint Luc, est associée à l'origine du christianisme.

13 En 1834, Athènes, une bourgade de 10.000 habitants à l'époque, devient la capitale de l'État. Il s'agit d'un choix révélateur, dans la mesure où la construction de l'identité grecque moderne est fondée sur deux références ${ }^{8}$ : la Grèce ancienne d'une part et Byzance d'autre part. En présentant la nation comme l'héritière directe de ces deux traditions, le nationalisme essaie de mettre l'accent sur la continuité de la civilisation grecque malgré l'occupation ottomane. En ce qui concerne l'église de Tinos, l'argument nationaliste est utilisé dès le début afin de l'imposer comme église emblématique de l'État. Les éléments historiques qui lui permettraient de se présenter comme héritière de la tradition byzantine lui faisant défaut, elle doit se contenter de marquer sa continuité avec la Grèce ancienne : son sanctuaire, construit lors de la révolution, l'a été pour une grande partie avec du marbre rapporté des ruines de Délos; il matérialise ainsi la rhétorique nationaliste de façon concrète. Étant donné que les Cyclades doivent leur nom au fait qu'elles forment un cercle autour de l'ancienne île sacrée de Délos, qui était non seulement un centre religieux mais aussi le centre de l'alliance maritime gouverné par les Athéniens, il devient clair qu'à l'instar d'Athènes qui devient la capitale du nouvel État, l'île de Tinos veut reprendre, dans un contexte chrétien cette fois, le rôle dominant de Délos.

14 Durant la première moitié du XIXe siècle, l'icône de Tinos avait déjà été visitée par le roi et les héros de la révolution. Ces événements décisifs pour son histoire ne figurent pourtant pas dans les manuels scolaires actuels. Avec la guerre de 1940, Tinos y gagnera sa place comme lieu de la première attaque italienne contre le peuple grec.

\section{L'icône - protectrice divine et la guerre de 1940}

15 Le 15 août 1940 (fête de la Dormition), un navire de guerre qui mouille aux environs de l'île de Tinos pour rendre hommage à l'icône est coulé par un sous-marin italien. Deux mois plus tard, le 28 octobre, la Grèce s'engage dans la Deuxième Guerre mondiale. Alekos Florakis (1990), un folkloriste originaire de Tinos, présente des documents de l'époque dans un livre ${ }^{9}$ édité récemment par l'Église de Tinos. Selon le corpus réuni, la guerre des Grecs contre les Italiens fut perçue à l'époque comme une "guerre sainte » destinée à restaurer l'honneur de la Vierge « insultée ». Dans le même esprit, les soldats grecs furent présentés comme des militaires-théologiens qui lisaient la Bible et portaient sur eux de petites reproductions de la Vierge de Tinos, tandis que les Italiens étaient des blasphémateurs qui portaient sur eux des images de femmes nues.

16 À l'inverse de la révolution de 1821, la guerre de 1940 n'a pas procuré à l'histoire nationale de héros individualisés. Pourtant, le navire de guerre coulé qui s'appelle « Elli » devient aussitôt un symbole personnifié : des affiches de l'époque montrent l'Elli au Ciel aux côtés de la Vierge de Tinos, qui protège les bateaux grecs pendant les combats navals contre les Italiens. Les textes de la même période expriment souvent le désir qu'un bateau italien, prisonnier de guerre, soit conduit jusqu'au port de Tinos, à l'endroit où l'Elli a été coulé, lors d'une fête du 15 août, pour être purifié avec de l'eau bénite et recevoir le nom de « sa victime ». L'Elli serait ainsi ressuscité et les âmes de ses morts pourraient trouver la paix, le navire ayant été purifié et «converti ». C'est après la fin de la guerre (qui se prolonge en Grèce par la guerre civile), en 1951, que ce désir se réalise et que le miracle 
de la résurrection de l'Elli se produit: un bateau italien donné en dommage de guerre arrive à Tinos et le même jour, à la même heure que celle du naufrage, est baptisé « Elli II ».

17 Le naufrage du bateau le 15 août, jour de la Dormition de la Vierge, constitue l'inverse symbolique de l'invention de l'icône de l'Annonciation. Cependant, cette fois-ci, le destin de l'icône et de la nation sont dissociés ou, tout au moins, l'un ne reflète pas l'autre, puisque les péripéties nationales n'affectent plus le statut de l'icône. Au contraire, le rôle de protectrice divine lui est attribué : elle est censée veiller sur les victoires grecques. Dans ce cas, entre la nation et l'icône s'interpose le bateau « sacrifié » dont le nom évoque facilement l'adjectif « hellénique ». Si la révolution de 1821 contribue à la renaissance simultanée de la nation soumise et de l'icône enterrée, la résurrection de l'Elli est un acte orchestré qui s'inscrit dans le même schéma. Dans ce cas, l'Église de Tinos et l'État grec réécrivent l'histoire non pas par l'interprétation et la manipulation des faits historiques, mais par la création d'un événement: au cours d'une cérémonie spéciale, ils « ressuscitent » le bateau coulé par les Italiens. Pour l'État, cet acte vise à reconstituer la continuité nationale. Pour Tinos, qui a passé cinq siècles sous occupation vénitienne (et un siècle seulement sous occupation ottomane) et dont une partie de la population reste toujours catholique, le "baptême " d'un bateau italien en "Elli II» est aussi sa " conversion » : le bateau était italien et catholique ; il devient grec et orthodoxe.

Aujourd'hui, au rez-de-chaussée de l'église de Tinos se trouve «le mausolée de l'Elli ${ }^{10}$ »: les os des victimes de ce naufrage y sont enterrés et certaines pièces du bateau y sont exposées aux yeux des pèlerins comme des reliques. De même que les livrets distribués s'approprient l'histoire nationale pour donner sens à l'invention de l'icône, de même « le mausolée de l'Elli » matérialise la sacralisation d'un événement politique et militaire.

Ce cas d'un «musée national » inscrit dans l'enceinte sacrée d'une église n'est pas unique en Grèce. Ainsi, des révolutionnaires de 1821 auraient révélé à leurs compagnons, au moment de leur mort, avoir caché leurs armes près de l'autel d'une église, pour que les Turcs ne puissent pas les trouver et les souiller. Ils auraient aussi demandé que leur fils aîné vienne les réclamer à leur majorité (Anagnostopoulos, 1984, p. 113). Très souvent également, ils ont offert leurs armes à une église en remerciement pour leur victoire. Ainsi, plusieurs monastères (surtout dans le Péloponnèse et en Grèce continentale) ont-ils aujourd'hui une collection d'armes de cette époque exposées dans des salles réservées à cet effet.

20 Selon Krzysztof Pomian (1987, p. 59), «(...) les musées prennent la relève des églises en tant que lieux où tous les membres d'une société peuvent communier dans la célébration d'un même culte. Aussi bien leur nombre croît aux XIX et XXe siècles, au fur et à mesure que grandit la désaffection des populations, surtout urbaines, pour la religion traditionnelle. Le nouveau culte qui se superpose ainsi à l'ancien, devenu incapable d'intégrer la société dans son ensemble, c'est celui dont la nation se fait en même temps le sujet et l'objet. » En Grèce, en revanche, les « deux cultes », religieux et national, sont complémentaires : autant les églises que les monastères continuent de revendiquer leur place dans la construction de l'histoire nationale. Les armes que les révolutionnaires mourants cachent près de l'autel d'un sanctuaire permettent en effet d'associer symboliquement le sacrifice d'un héros pour sa patrie et le sacrifice perpétuel du Christ pour la rédemption de l'espèce humaine.

21 Le «mausolée de l'Elli » réalise aussi le lien entre culte religieux et national, mais d'une façon un peu différente. Alors que les musées des églises et monastères évoquent le 
sacrifice individuel des héros de la révolution, le second met l'accent -à l'instar des «Monuments aux morts» construits par l'État - sur le sacrifice d'hommes anonymes. D'autre part, ce mausolée est, en réalité, plus qu'un «Monument aux morts », puisque l'Elli constitue l'équivalent symbolique de la nation elle-même ainsi que son homonyme. Avec ce mémorial, l'église de Tinos ne contient pas seulement les restes matériels qui commémorent un événement national; elle prétend plutôt contenir la métaphore matérialisée d'un État momentanément écroulé. Or, dans les affiches de l'époque, c'est la Vierge de Tinos qui amène l'Elli (et, en même temps, la nation grecque) au Ciel, et c'est l'église de Tinos avec ce « mausolée » qui fait de cette nation un martyr de l'histoire.

Entre l'histoire nationale et l'histoire de l'icône s'interpose pourtant l'histoire locale de l'île de Tinos. Comme Jill Dubisch (1995, p. 122) ${ }^{11}$ le constate, " pour la plupart de ses habitants, l'invention de l'icône marque le "début" de l'histoire de Tinos ". Je me propose donc maintenant d'examiner comment les brochures de Tinos présentent le destin de l'île comme dépendant de l'Institution de Tinos.

\section{La société locale, l'Institution et l'État grec}

L'ouvrier qui trouva l'icône de Tinos la fendit accidentellement en deux. Mais les femmes nobles de l'île, quand elles vinrent rendre hommage à l'icône pour la première fois, placèrent sur elle leurs bijoux les plus précieux afin de cacher la fente. Aujourd'hui on ne voit plus la «blessure» provoquée par la pioche, puisque les bijoux recouvrent entièrement la surface peinte. Ainsi, ce sont les bijoux offerts par les femmes de Tinos qui ont « réparé » le dommage fait à l'icône et qui l'ont « reconstruite ». Ce premier temps de l'invention est donc marqué par un geste de la communauté locale, qui offre ses richesses à l'icône. En retour, ce sera à l'icône d'assurer un présent et un futur prospère à l'île de Tinos.

24 En janvier 1825, le conseil municipal qui a dirigé les opérations pour le déterrement de l'icône dépose un «testament » qui jette les bases de la direction administrative actuelle et en définit les règles. Selon ce " testament ", l'église qui héberge l'icône miraculeuse ne constitue ni une église paroissiale ni un monastère, mais une Institution chargée de payer les professeurs et de maintenir les écoles créées par le conseil. L'Église de Tinos adopte ainsi un profil plus éducatif que religieux; son rôle en ce qui concerne l'éducation au niveau local lui permet de répandre le culte de son icône.

Cette Institution a ses propres ressources économiques et ses propres frais. Elle est contrôlée directement par l'État grec, à la différence des autres sanctuaires du pays qui sont gérés par le Synode ecclésiastique; plus précisément, elle dépend de deux ministères, celui de l'Éducation Nationale et des Cultes, et celui de l'Économie. Les popes qui servent la messe sont les employés de l'Institution et ils ne participent pas à sa direction administrative. Elle est dirigée par un conseil de dix membres, dont six sont élus, à condition qu'ils soient originaires de l'île et orthodoxes. Le président du conseil est, honoris causa, l'évêque de l'île. Les trois autres membres sont le directeur du lycée, le juge et le responsable du service fiscal de lîle, donc les représentants locaux de l'État. Ce statut exceptionnel fait de l'Église de Tinos la seule église grecque qui ne soit pas subordonnée au clergé, mais directement à l'État grec - ce qui marque la spécificité de son association au pouvoir politique. 

l'Institution comme un pionnier qui a pris des initiatives sociales au niveau local bien avant l'État grec. Selon eux, les écoles de l'île fonctionnaient dès 1825, alors qu'il n'y avait pas encore un système d'éducation planifié dans le reste de la Grèce. De même, l'Institution donnait des retraites à ses employés, professeurs et clercs, quand la politique sociale de l'État n'était pas encore institutionnalisée. Les îles grecques, aujourd'hui, ont encore des problèmes d'insuffisance en eau potable, surtout pendant l'été. L'Institution, en revanche, a réalisé depuis longtemps de grands travaux hydrauliques, qui permettent à la ville de Tinos d'avoir suffisamment d'eau pour les habitants et les 200.000 pèlerins qui débarquent chaque année sur l'île. Ainsi, l'Institution est présentée comme le substitut de la providence étatique en plusieurs occasions, et comme le seul à pouvoir assurer un bon niveau de vie aux habitants de l'île. Parmi les œuvres que l'Institution a entreprises, les brochures citent la construction du port, l'installation de l'électricité dans tous les villages et, plus récemment, la préparation technique pour la construction d'un aéroport. autarcique : prospère au niveau économique, juste au niveau social et bénie par la grâce de l'icône miraculeuse qu'elle héberge. L'Institution imite les structures étatiques, mais en même temps elle est présentée comme l'incarnation d'une perfection que l'État grec n'a jamais pu atteindre.

Nous avons vu que l'icône de Tinos subit l'agressivité des musulmans deux siècles avant la nation grecque; de façon analogue, elle «se libéra » aussi quelques années avant elle. Pourtant, dès que l'État grec se forma pour gérer le «destin national ", l'icône de Tinos n'apparut plus comme le thermomètre infaillible de ce destin. D'une certaine manière, elle se situe au-dessus de l'État, puisque ce dernier peut subir des humiliations politiques provenant des puissances internationales, des défaites militaires et des scandales en ce qui concerne la vie politique à l'intérieur. Ces moments de crise ne touchent plus l'icône. Ses péripéties finissent avec son invention, tandis que les péripéties de la nation continuent. Ainsi, elle reste détachée du cours de l'histoire ou, plus précisément, elle n'y participe plus que comme protectrice divine. Pourtant, il apparaît que ce n'est pas seulement l'icône que son statut surnaturel place au-dessus des événements historiques, à l'opposé d'un État qui subit toutes les perturbations de cette histoire; c'est aussi l'île entière qui est présentée comme le modèle réussi, autant céleste que terrestre, auquel devrait se conformer l'État grec.

Les imprimés ne soulignent pas seulement le rôle de l'Institution au niveau local; elle a aussi un rôle national. Ainsi, Tinos accueillit-elle des réfugiés originaires d'autres régions de la Grèce durant la révolution de 1821. Depuis lors, l'Institution soutient matériellement l'Etat aux moments des guerres, des calamités naturelles et des crises nationales. En Grèce, c'est l'État qui finance les églises paroissiales et qui paie les popes. Selon les brochures, l'Institution de Tinos arrive à le remplacer même en ce qui concerne le domaine ecclésiastique. Par exemple, c'est l'Institution qui donne le plus d'argent après la fin de la guerre civile pour la restauration des églises endommagées partout en Grèce. Étant donné que l'administration de l'Institution est présentée comme « constituant ellemême un miracle ", il devient clair que c'est plutôt l'État qui s'appuie sur l'Institution que le contraire. L'Institution, bien sûr, dépend officiellement de cet État ; mais sa prospérité, qui se concrétise dans le progrès matériel de l'île, le caractère miraculeux de ses ressources financières et son rôle actif au niveau national contribuent à la situer au- 
dessus de lui. Paradoxalement, l'Institution de Tinos se mesure principalement à l'État et non pas aux autres institutions religieuses.

Jusqu'ici nous avons analysé comment l'Église de Tinos se présente actuellement au travers des brochures destinées aux pèlerins. Après avoir étudié l'histoire légendaire de l'icône et le fonctionnement idéalisé de l'Institution, il est temps maintenant d'examiner ce que Tinos représente pour les pèlerins et ce que disent leurs pratiques du sens de la dévotion à l'icône.

\section{Les pratiques dévotionnelles : de la nation à l'individu}

31 Les brochures de Tinos ne parlent pas des pratiques dévotionnelles. Elles se contentent de présenter certains miracles dont le plus récent date du 29 juillet 1980 : alors que sa femme est en danger après un accouchement difficile, le mari implore le secours de la Panayia de Tinos, qui sauve la femme. En signe de gratitude le couple abandonne sa vie en Crète et vient habiter à Tinos. L'île est ici présentée comme une source de vie : tout étranger peut y venir (et dans les cas extrêmes y rester) pour retrouver la santé et s'assurer les multiples bénéfices du contact avec l'icône.

Le même esprit caractérise le livre d'un écrivain grec, Georgios Drossinis (1992), qui assiste à la fête du 25 mars 1883 sur l'île de Tinos. Le texte décrit la transformation de la ville de Tinos pendant cette période festive: les habitants louent leurs maisons aux pèlerins les plus riches; les pèlerins les plus pauvres dorment dans les rues de la ville. Les habitants mettent en location leurs boutiques, leurs cafés et leurs commerces, et ainsi, durant ces trois ou quatre jours l'île devient un centre de commerce pan-hellénique. On y trouve des produits qui viennent de toute la Grèce, mais aussi d'Asie Mineure, d'Égypte et de toutes les colonies grecques importantes. Cette concentration spatiale et temporelle ne concerne pas seulement les produits ; les pèlerins eux aussi représentent une multitude de régions, de dialectes et de costumes traditionnels.

Quant aux pratiques dévotionnelles, Drossinis cite d'abord l'habitude des gens de porter leur nouveau-né à Tinos pour le faire baptiser devant l'icône de la Vierge. Après avoir expliqué que devenir le parrain d'un tel enfant constitue un grand honneur, il décrit la manière dont les pèlerins se pressent autour des enfants, puisque le premier dévot qui arrive à en saisir un dans l'église de Tinos devient, selon cette coutume, son parrain. Une autre pratique concerne les femmes enceintes qui arrivent sur l'île pour accoucher sous la protection de la Vierge. L'écrivain exprime sa surprise devant une pratique pénitentielle inconnue de lui, quand il voit une pèlerine marcher pieds nus du port jusqu'à l'église. Cependant, Drossinis ne se contente pas de parler de la piété individuelle; il commente aussi l'enthousiasme nationaliste de ces pèlerins qui, pour une bonne partie, ne sont pas citoyens grecs, mais qui attendent toujours le rattachement de leur région à la Grèce. Pour ces pèlerins, être sur l'île de Tinos durant quelques jours signifie « respirer l'air de la liberté pour un moment » (Drossinis, 1992, p. 55). L'écrivain critique même l'État grec qui ne se soucie pas de donner à cette fête religieuse un caractère national afin d'animer les esprits et de faire connaître ses revendications territoriales.

Ce récit laisse apparaître clairement que l'île de Tinos représentait à l'époque le «cœur sacré » de la Grèce. Plusieurs hommes et femmes, citoyens ou non du pays, s'y rendaient pour y donner naissance, y faire baptiser leurs enfants, y vendre leurs produits et accomplir leurs vœux; mais aussi pour se sentir membres de la communauté nationale. 
Dans ce cas, une fête religieuse offre le cadre de l'expression des sentiments nationalistes; les pèlerins sont aussi des patriotes. Selon Drossinis, ce rassemblement n'est pas dû (comme il le faudrait) à la propagande nationaliste de l'État. Pourtant, si mon hypothèse de départ est exacte, l'Institution de Tinos s'est appuyée sur l'idéologie nationaliste pour assurer sa place comme « sanctuaire national » du pays. Le cas de Tinos nous oblige ainsi à marquer la distinction entre un nationalisme étatique et un courant nationaliste provenant de la base qui trouve son expression dans le cadre religieux.

Selon la pratique présentée par Drossinis, un simple dévot inconnu peut devenir parrain d'un enfant qui vient à Tinos pour être baptisé. Il semble que le simple fait d'être sur l'île et d'assister aux cérémonies religieuses suffise pour faire d'un étranger le parent spirituel d'un enfant, puisque ce parrainage est désigné et béni par l'icône de la Vierge. Tinos devient ainsi le lieu où les Grecs de différentes régions se rencontrent, font la fête ensemble et éventuellement s'unissent entre eux par des relations spirituelles. Selon cette image idyllique de cohérence sociale et d'homogénéité, la nation grecque est présentée comme une grande famille pieuse.

Florakis (1990, p. 162) cite un autre cas de pratique dévotionnelle qui obéit au même imaginaire. Les milliers d'ex-votos déposés à Tinos représentent le plus souvent une partie du corps, une personne entière, un bateau ou l'accomplissement d'un miracle. Mais il en est aussi qui représentent l'île de Chypre. Les plus anciens d'entre eux furent offerts à l'icône en remerciement de la libération de l'île du joug anglais ; les plus récents (c'està-dire après 1974), pour demander à la Vierge la libération de la partie sous occupation turque. Dans ce cas, le vœu dépasse l'individu ou le cadre familial et embrasse la communauté nationale ${ }^{12}$. Par ce geste, le Chypriote anonyme traite sa nation comme une partie de son propre corps ou un membre de sa famille touchés par la maladie. Sur ce point, il faut revenir au temps de l'invention de l'icône de Tinos. Selon les brochures, à l'instar de la nation grecque en rébellion mais toujours occupée par les Ottomans, les fouilles entreprises par la communauté de l'île ne donnèrent pas de résultats immédiats; l'icône resta enterrée et les habitants découragés arrêtèrent les opérations. La peste s'abattit alors sur Tinos. Les fouilles recommencèrent et, une fois l'icône découverte, elle fut portée en procession dans toute l'île: la peste s'arrêta miraculeusement. Dans ce cas, entre la nation soumise et l'icône enfouie, s'interpose le "corps" malade de la communauté locale, de telle façon que la «libération » de l'icône préfigure autant l'émancipation nationale que la guérison collective du « corps social » au niveau local.

Ce type de légendes est très courant en Grèce et vise toujours à légitimer la position primordiale de l'icône patronale d'un village, d'une ville ou d'une île ${ }^{13}$. De façon analogue, le Chypriote qui offre l'ex-voto de son île à la Vierge de Tinos la reconnaît par ce geste comme icône « nationale » et pan-hellénique. C'est à cette icône de procurer le bien-être aux moments critiques au corps d'un individu, au « corps » d'une localité ou au «corps » d'une nation ${ }^{14}$.

39 Le « corps » de la nation grecque est représenté dans l'église de Tinos par le "mausolée de l'Elli»: au centre de ce "mausolée », se trouve une statue de marbre de style néoclassique figurant une femme couronnée, avec des ailes d'ange et qui porte une branche de laurier. Devant le portail de l'enceinte sacrée de Tinos, nous rencontrons une autre statue, plus récente. Elle est en bronze, de style réaliste, et représente une femme habillée comme une paysanne, avec une robe longue et un foulard sur la tête, qui marche à genoux et tend la main vers l'église. Cet autre «corps», moins glorieux, est celui du 
pèlerin: en effet, le pèlerinage à Tinos est actuellement connu pour son caractère sacrificiel ; marcher à genoux du port à l'église constitue la pratique dévotionnelle la plus courante.

40 La position de ces deux monuments (le monument « nationaliste » au centre de l'église, le monument qui commémore les pratiques sacrificielles - et qui les rend aussi esthétiques et normatifs - à la périphérie) montre bien comment Tinos se définit d'emblée comme "icône nationale »: elle protège principalement la nation et, à un deuxième niveau, chaque individu séparément. Il nous faut pourtant poser la question de savoir si elle constitue, pour les Grecs d'aujourd'hui, un symbole religieux national comme elle le prétend.

\section{Les contradictions de la modernité}

41 Aujourd'hui, c'est surtout pendant le mois d'août que les pèlerins affluent à Tinos. Durant toute cette période, de nombreux baptêmes ont lieu dans une chapelle au rez-de-chaussée de l'église; elle est placée entre celle qui commémore l'invention de l'icône et le « mausolée de l'Elli ». Les baptistères qui se trouvent entre ces deux pôles transforment la symbolique qui leur est attachée en réalité actuelle : chaque enfant se trouve immergé, puis sorti de l'eau, comme l'icône et le bateau «Elli ». Par ailleurs, l'emplacement des baptistères fait qu'un enfant à Tinos est baptisé en même temps orthodoxe et "grec ", puisque son baptême le met en relation directe avec la formation de l'État (préfigurée, selon la brochure, par le déterrement de l'icône) et le «mausolée de l'Elli». L'aménagement de l'espace contribue à investir ce sacrement d'un sens nationaliste. Pourtant, le baptême d'un enfant à Tinos constitue, pour les parents, soit l'accomplissement d'un vœu personnel dans le cas d'enfants en mauvaise santé, soit un acte dévotionnel destiné à mettre l'enfant sous la protection de la Vierge; dans les deux cas, aucune connotation nationaliste ne caractérise aujourd'hui ces baptêmes, du moins dans la conscience des dévots. Il semble qu'actuellement une distance se creuse entre la symbolique nationaliste de ce lieu et la perception qu'en ont les pèlerins.

Prenons comme exemple le cas récent d'un jeune Athénien de 28 ans, non pratiquant, qui ne sait même pas ce qu'est un ex-voto, et qui a visité l'île de Tinos à deux reprises; la première fois, amené par sa mère, la seconde fois, amené par la «mère-patrie ", selon l'expression grecque. L'histoire commence avec sa mère qui, étant enceinte de lui, fait le vœu de le baptiser à Tinos, si sa grossesse et son accouchement se déroulent normalement. Aujourd'hui, elle raconte encore les difficultés qu'elle a rencontrées quand elle a voulu accomplir son vœu : le voyage à Tinos était pénible, il faisait très chaud et il était impossible de trouver une chambre à louer sur l'île ; puis, le bébé est tombé malade et a failli mourir. Le récit de la mère (qui n'est pas spécialement pratiquante) présente l'accomplissement du vœu comme un sacrifice important et rappelle, dans une certaine mesure, les péripéties de la Sainte Famille avant la naissance du Christ. Quelques années plus tard, le jeune homme fait son service militaire dans la marine. Son bateau se rend à Tinos pour la fête de l'Annonciation, qui est aussi une fête nationale. Là, l'équipage du bateau (dont le jeune homme fait partie) rend hommage à l'icône de Tinos et l'escorte pendant la procession. Ainsi, ce jeune Athénien fut d'abord pèlerin, puis membre de la délégation militaire. Dans les deux cas, ces rôles lui furent attribués en dehors de sa volonté; par ailleurs, il convient de souligner que le premier acte relève de la piété 
individuelle, alors que le second est une pratique cérémonielle instituée par le pouvoir étatique.

On ne sait pas exactement à quelle date l'État a commencé à envoyer des représentants à Tinos, mais sûrement avant 1940, puisque le bateau de guerre de l'Elli y était à ce titre au moment où il fut attaqué. En Grèce, il existe deux fêtes nationales : la première est le 25 mars ; la deuxième, le 28 octobre, commémore le refus du Premier ministre de satisfaire la demande de reddition envoyée par l'Italie en 1940. Sur l'île de Tinos, les trois fêtes les plus importantes sont celle de la Dormition de la Vierge (15 août), celle de l'Annonciation (25 mars) et celle qui commémore l'invention de l'icône (30 janvier). Les deux premières correspondent plus ou moins aux fêtes nationales, puisque la fête de la Dormition est associée au naufrage de l'Elli qui préfigure l'entrée en guerre du pays contre l'Italie. Actuellement, ce jour est aussi la fête de l'Armée. Chaque année, à ces deux occasions, une délégation du gouvernement et de l'armée arrive sur l'île afin de représenter l'État pendant les festivités religieuses.

La demande que l'écrivain grec, Georgios Drossinis, avait formulée à la fin du XIXe siècle fut alors rapidement satisfaite: mais on peut se demander si la présence institutionnalisée de l'État sur l'île de Tinos suffit aujourd'hui à donner à la fête religieuse un sens national? Lieu de pèlerinage à caractère thérapeutique, mais aussi lieu sacré directement lié au politique et au «destin national », l'île cycladique de Tinos essaie actuellement d'en gérer les contradictions: comment être en même temps l'icône emblématique de la nation et l'icône qui procure le mieux des « miracles individuels »? Si à la fin du siècle dernier, selon le texte de Drossinis, ces deux rôles étaient compatibles, à la fin de notre siècle il semble qu'ils ne le soient plus. Après la Deuxième Guerre mondiale, les frontières du pays sont définitivement fixées et l'État ne donne plus la même importance aux revendications territoriales; dorénavant, l'accent est mis sur la modernisation du pays. L'Église de Tinos ne peut pas se conformer à ce modèle de modernisation inspirée par des réalités occidentales et laïques ${ }^{15}$. Devant un tel déplacement de la politique étatique, Tinos ne peut que mettre en valeur l'esprit novateur des œuvres que l'Institution entreprend au niveau local ; elles ne suffisent pas cependant pour lui assurer une place comme agent du progrès au niveau national. Ainsi, Tinos représente plus aujourd'hui un passé traditionaliste qu'un futur progressiste.

Même si les brochures essaient de placer l'icône hors du temps historique en lui attribuant le rôle de protectrice divine, il semble que ce rôle ne prenne valeur qu'aux moments critiques, tels que les guerres. Pendant les périodes de paix et de développement économique, l'efficacité de l'icône de Tinos se limite inévitablement à la protection de l'intégrité corporelle des individus. En effet, la présence de l'État sur l'île ne vise qu'à commémorer deux guerres, deux événements cruciaux de la mémoire nationale. Cette commémoration souligne et reconnaît le rôle de Tinos dans le passé, et non dans le présent.

46 Ce sont les classes sociales moyennes ou basses qui fréquentent principalement l'île de Tinos. Il s'agit d'un pèlerinage populaire dont le caractère irrationnel et le sentimentalisme sont critiqués par les classes supérieures comme étant anachroniques et embarrassants. Il est clair que pour elles, cette église n'est pas le lieu religieux emblématique de la Grèce contemporaine. Tinos semble donc menacée par l'ambivalence de son statut. Si à un certain moment c'est sa popularité qui a forcé l'État à la reconnaître comme "sanctuaire national», cette popularité contribue actuellement, dans une certaine mesure, à la rendre inapte à conserver ce rôle. Cette ambiguïté crée pendant les 
fêtes la contradiction visible entre, d'une part, les membres du gouvernement et les militaires, sérieux dans leurs uniformes, et d'autre part, les pèlerins qui se traînent sur le sol pour accomplir leurs voeux; les premiers sont là pour commémorer le passé de la nation, tandis que les seconds confient leur présent et leur avenir personnel à l'icône de Tinos.

Aujourd'hui, la place de Tinos comme lieu privilégié de pèlerinage semble bien assurée. Même les autres églises plus anciennes, régionales ou locales, du territoire qui hébergent une icône miraculeuse l'utilisent comme point de référence: une femme qui n'a pas trouvé la guérison à Tinos, est guérie par l'icône patronale de telle île ; telle église était un lieu très fréquenté avant que Tinos ne devienne si célèbre. Pourtant, Tinos continue d'utiliser l'idéologie nationaliste au travers de sa brochure. Là aussi, comme dans le cas du baptême des enfants, il y a une distance entre le mode de présentation de l'église et la perception des pèlerins. Actuellement, la plupart d'entre eux ignorent que l'icône fut trouvée pendant la révolution grecque. Ils ne savent pas que l'Institution de Tinos constitue la seule église du pays qui dépend directement de l'État grec et non pas de l'Église. Ces pèlerins ne s'intéressent pas à l'historique de l'icône, mais à son efficacité miraculeuse.

En examinant l'histoire, le statut administratif et les pratiques concernant l'église de Tinos, nous avons essayé de définir son importance comme lieu de culte dans le passé proche et le présent. Tinos s'est appuyée dès le début sur l'idéologie nationaliste, sur la presse et sur le système éducatif qu'elle a financé au niveau local, pour s'imposer comme le lieu de pèlerinage principal de la Grèce contemporaine : son icône est sortie de terre en même temps que cette terre redevenait grecque; son église a été construite avec du marbre de Délos; elle a été visitée par les héros de la révolution ainsi que par les noncitoyens du pays dans l'espoir que leur région soit rattachée à la Grèce. Mais le passage du pays du stade de la construction nationale à celui de la modernisation a affecté Tinos et sa place dans la société grecque. L'île sacrée s'est trouvée reléguée du centre des événements politiques à la périphérie de la piété individuelle.

\section{BIBLIOGRAPHIE}

* Ouvrages en grec.

*ANAGNOSTOPOUlos Ioannis S, La mort et "l'univers souterrain » (kato kosmos) selon la poésie populaire. L'eschatologie de la poésie populaire, Athènes, Potamitis Press, 1984.

CHRISTIAN William, Local Religion in Sixteenth Century Spain, Princeton, Princeton University Press, 1981.

DANFORTH Loring M, The Macedonian Conflict. Ethnic Nationalism in a Transnational World, Princeton, Princeton University Press, 1995.

*DRossinis Georgios, Trois jours sur l'île de Tinos, Athènes, Erinni, 1992, (première édition en 1883). 
DUBISCH Jill, "Golden oranges and silver ships: An interpretive approach to a greek holy shrine" in Journal of modem greek studies, $\mathrm{n}^{\circ}$ 6, 1988, pp. 117-134.

DUBISCH Jill, In a Different Place. Pilgrimage, Gender, and Politics at a Greek Island Shrine, Princeton, Princeton University Press, 1995.

* FloRAKIs Alekos E, La Panayia de Tinos pendant la guerre de 1940. Commémoration du cinquantenaire du naufrage de l'Elli et du commencement de la guerre, Athènes, édition de l'Institution sainte et panhellénique de l'Evangelistria Tinos, 1990.

GILLET Olivier, Religion et nationalisme. L'idéologie de l'Eglise orthodoxe roumaine sous le régime communiste, Bruxelles, éditions de l'Université de Bruxelles, 1997.

HERZFELD Michael, Ours Once More : Folklore, ldeology, and the Making of Modem Greece, Austin, Universtity of Texas Press, 1982.

HERZFELD Michael, Cultural Intimacy. Social Poetics in the Nation-State, New York-Londres, Routledge, 1997.

*KORnAROS Elephterios N, La Panayia de Tinos. L'invention de l'icône sainte et les miracles de sa grâce, Athènes, 1968.

POMIAN Krzysztof, Collectionneurs, amateurs et curieux. Paris, Venise : XVI-XVIII siècle, Paris, Gallimard/N.R.F., 1987.

SPANAKI Marriana, "Byzantinism in contemporary icon production", in Byzantine and Modern Greek Studies, n¹7, 1993, pp. 163-169.

\section{NOTES}

1. En Grèce, la guerre d'indépendance de 1821 contre les Ottomans est appelée « révolution ».

2. Le terme « invention » est utilisé ici dans son sens latin de « découverte ».

3. VITALIS Filaretos, A, L'évêque de Tinos, Gabriel, pp. 49-51, note 40, Athènes, Éditions de l'Église de Preveza, 1957.

4. Pour retracer l'histoire nationale et l'histoire de l'icône de Tinos, je m'appuierai principalement sur les manuels scolaires récents et sur la brochure de 39 pages, éditée en 1995 par l'Institution de Tinos et illustrée de photographies en couleur, qui est actuellement donnée aux pèlerins. Cette brochure résume de façon significative les nombreux livres qui présentent l'histoire de l'icône - histoire qui fut d'abord écrite par Dionissios Pirgos et dont le livre avait déjà été réédité neuf fois en 1865 . Une nouvelle brochure est éditée chaque année : on y retrouve le même corpus narratif, avec des petites variations que je ne juge pas nécessaire d'analyser ici.

5. Dans les deux cas, c'est l'esprit collectif et la conscience d'appartenance à un groupe qui sont mis en valeur autant par les manuels d'histoire que par les brochures de Tinos.

6. C'est le nom courant de la Vierge en grec et signifie « la toute sainte».

7. Présentée dans le livre de Kornaros (1968, p. 24), un religieux, membre du conseil actuel qui dirige l'Institution de Tinos.

8. Voir l'analyse de Michael HERzFELD (1982).

9. Ce livre qui s'adresse non pas à une communauté de dévots, mais à la communauté scientifique, propose une lecture religieuse de l'histoire nationale.

10. Son nom (" mausolée ») évoque apparemment la Grèce antique et la continuité nationale.

11. Jill DUBISCH est la première anthropologue qui étudia le pèlerinage de Tinos et son caractère nationaliste. Voir aussi son article (1988).

12. Sur la relation entre patriotisme et orthodoxie en Roumanie, cf. Olivier GILLET (1997). 
13. Sur les vœux collectifs en ce qui concerne le monde catholique, cf. William CHRISTIAN (1981).

14. Sur l'utilisation des métaphores du corps par le nationalisme, cf. Michael HERZFELD (1997).

15. Dans les années cinquante, apparaît aussi le courant du «byzantinisme », selon l'expression de Marianna SPANAKI (1993). La tradition byzantine est réévaluée et l'art byzantin est recommandé comme seule forme acceptable pour la décoration des églises. Dans ce cadre, apparaît plus tard le mouvement intellectuel des "néo-orthodoxes" qui essaie de définir l'alternative d'une modernisation inspirée par la spiritualité orthodoxe. Tinos, de son côté, continue de se présenter selon le modèle nationaliste mis en valeur par sa brochure, sans tenir compte de ces changements.

\section{RÉSUMÉS}

Le sanctuaire de l'île cycladique Tinos, est le lieu de pèlerinage le plus important de la Grèce contemporaine. Il a assis sa réputation en articulant l'histoire de son icône miraculeuse à l'Histoire Nationale : la Vierge qui y est vénérée aurait été découverte au moment où les Grecs commençaient à lutter pour chasser les Ottomans, qui dominaient alors le pays depuis quatre siècles; le sanctuaire abrite un "mausolée " dédié aux marins-soldats de l'Elli, un navire de guerre coulé par les Italiens lors de la Seconde Guerre mondiale. Le culte de cette icône, cependant, tend à perdre, aujourd'hui, sa connotation nationaliste: les Grecs se rendent actuellement à Tinos pour demander la satisfaction de vœux d'ordre individuel et non plus par patriotisme. Cet article analyse la tension entre ces fonctions religieuses et politiques.

The shrine of Tinos, an island of the Cyclades, is the most important pilgrimage site of modern Greece. Its reputation has been based on the association of the history of its miracle-working icon with the National History: the icon of the Virgin Mary has been discovered during the Greek struggle for independence after four centuries of Turkish rule; the shrine shelters a "mausoleum" commemorating the sinking of the battleship Elli by the Italians during the Second World War. Nevertheless, the cult of this icon tends to lose, nowadays, its nationalist connotation: Greeks visit the island of Tinos in order to demand the satisfaction of a personal wish, and not by patriotism. This paper analyses the tension between these political and religious functions.

El santuario de Tinos, una de las islas Cíclades, es el lugar de peregrinación más importante de la Grecia contemporánea. Su reputación se basa en la articulación de la historia de su ícono milagroso con la historia nacional: la Virgen que se venera habría sido descubierta en el momento en que los Griegos comenzaban a luchar para expulsar a los Otomanos, que dominaban enfonces el país desde cuatro siglos atrás. El santuario comprende un "mausoleo" dedicado a los marinos-soldados del Elli, una nave de guerra hundida por los italianos en la Segunda Guerra Mundial. El culto de este ícono, sin embargo, tiende a perder hoy en día sus connotaciones nacionalistas : los Griegos van actualmente a Tinos para pedir deseos de orden individual y no ya ligados al patriotismo. Este artículo analiza la tension entre estas funciones religiosas y politicas. 
AUTEUR

KATERINA SERAÏDARI

Centre d'Anthropologie - Toulouse 